\title{
THESEN
}

\section{Balancieren zwischen Stabilität und Instabilität}

\section{Andreas Strunk}

Die Sozialwirtschaft befindet sich in der Legitimationskrise. Wie die einzelne Organisation diese Zeit bewältigt, hängt ab vom Umgang der Verantwortlichen mit Stabilität und Instabilität. Ein kluges Veränderungsmanagement setzt auf die Organisation von Wertkonflikten und die Beteiligung aller.

$\mathrm{D}$ er Autor beschäftigt sich seit Anfang der 1970erJahre praktisch und wissenschaftlich mit Vorhaben des Veränderungsmanagements in der Sozialwirtschaft. Er setzt vor allem auf ein methodisches Konzept der Selbstorganisation.

Hier ist er stark beeinflusst von dem Bremer Wissenschaftler Prof. Dr. Peter Kruse, mit dem er 1994 Konzepte zu einer Entinstitutionalisierung einer »Großpsychiatrie « entworfen hat. Peter Kruse legte zu dieser Zeit die Grundlage für sein Konzept des Balancierens zwischen Stabilität und Instabilität als Modell einer modernen Unternehmensentwicklung. (1) Dieses Konzept ist inzwischen von Peter Kruse erfolgreich weiterentwickelt worden und wird in dem vorliegenden Heft der Zeitschrift SOZIALwirtschaft erläutert.

Die 30 Thesen von Andreas Strunk spiegeln sowohl seine eigenen Erfahrungen als auch die Erfahrungen und Erkenntnisse von Peter Kruse wieder, wie diese von ihm in dem auf Seite 13 besprochenen Buch »Next Pracitce. Erfolgreiches Management von Instabilität. Veränderung durch Vernetzung « beschrieben werden.

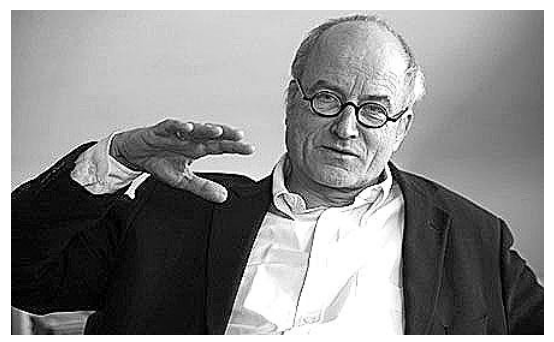

Prof. Dr. Andreas Strunk ist Hochschullehrer an der Fachhochschule Esslingen und vertritt dort die Fächer Sozialpädagogik und Organisationswissen für die Soziale Arbeit. Er ist Gesellschafter der GISAmbH und konzentriert sich dort auf Vorhaben der Organisationsentwicklung und Führungskräfteberatung.

E-Mail prof.strunk@t-online.de
1 Veränderungsmanagement organisiert einen Ent1 - wicklungsprozess in einer Organisation mit dem Ziel, die Leistungsfähigkeit dieser Organisation angesichts neuer Kontextbedingungen zu optimieren. Der Entwicklungsprozess muss geplant, durchgeführt und evaluiert werden.

2 Die Sozialwirtschaft befindet sich in einer Erneue• rungskrise, von der man schlecht abschätzen kann, ob es sich um eine »schöpferische Zerstörung « oder um eine »Zerstörung des Schöpferischen « handelt. Ein schöpferischer Ausweg aus der Krise hängt auch von der Qualität des Veränderungsmanagements in der Sozialwirtschaft ab.

2 Da Veränderungsmanagement sorgfältig geplant 3 werden muss, wird qualifiziertes Planerwissen benötigt. Die Ausgangslage in der Sozialwirtschaft ist hoch komplex. Das bedeutet für Planung und Durchführung von Veränderungsmanagement, dass die Organisation von Wertkonflikten und die Beteiligung von Betroffenen im Vordergrund stehen müssen.

$\Delta$ Wenn Veränderungsmanagement erfolgreich sein • soll, muss man auf das organisationale Selbstverständnis der Organisation achten, wenn man nicht Schiffbruch erleiden will. Es gibt viele systemisch bedingte Unverträglichkeiten. So wird man mit einer Strategie der Selbstorganisation in Organisationen, die streng auf Hierarchie setzen, wenig ausrichten können.

5 Es gibt nicht »die Sozialwirtschaft«. In der Sozialwirt5 . schaft gibt es unterschiedliche Welten und Wirklichkeitskonstruktionen. Das Management der Sozialwirtschaft wird sich einer neuen Professionalisierung stellen müssen. Es geht um die Kunst des Balancierens zwischen Stabilität und Instabilität in jedem organisationalen Einzelfall. Management von Instabilität muss mit Unsicherheit umgehen können. Hier ist eine entsprechende organisatorische Intelligenz nötig. Das Verständnis von und das bewusste Umgehen mit Instabilität gibt eine gewisse Sicherheit in der Unsicherheit.

6 In der Organisation müssen die Personen und Grup- pen identifiziert werden, die an alten Mustern festhalten wollen. Hier ist zu fragen, ob sie irritierbar sind. Denn sie werden möglicherweise auf notwendige - und phasenweise bewusst eingeführten - Irritationen lösungsorientiert reagieren müssen.

7 Zur Kunst des Balancierens zwischen Stabilität und - Instabilität gehört auch die Identifikation von Bereichen in der Organisation, die weiterhin von Stabilität be- 
stimmt sein müssen. Zu viel Instabilität kann eine Organisation zerstören. Insofern ist Achtsamkeit im Umgang mit unverzichtbaren Stabilitätsanteilen der Organisation notwendig.

Q Aufgrund des sehr hohen und zum Teil unübersicht8. lichen Innovationsdruckes, der auf das System der Sozialwirtschaft einwirkt, ist es oft nicht prognostizierbar, welche organisationale Antwort in dem Unternehmen »richtig« oder »falsch « ist. Insofern weiß niemand so recht, wohin die Reise geht.

Q »Faszination ist wohl die unerschöpflichste und posi• tivste Quelle menschlicher Kreativität und Veränderungsbereitschaft. Sie besteht im Kern aus der Mischung von Irritation und Neugier." (Peter Kruse) Es ist deshalb sinnvoll, auf der Führungsebene eine Vision für die Fortentwicklung der Organisation zu entwickeln. Sie wird als emotionale Basis für das Veränderungsmanagement dienen können.

10. Auf der Führungsebene wird man die Spreu vom - Weizen trennen müssen, indem Normstrategien festgelegt werden. Denn: »Leistungsträger, die eine Vision nicht teilen, sind keine Querdenker, sondern Quertreiber. Sie zerstören die Glaubwürdigkeit der Veränderung. «(Peter Kruse)

11 In der Organisationsentwicklung gibt es keine iso- lierte Selbstorganisationsmöglichkeit für Mitarbeiterinnen und Mitarbeiter. Ihre Selbstorganisation ist eingebunden in strukturelle Vorgaben. Die Entwicklung und Pflege dieser Vorgaben ist Aufgabe der Führungsmannschaft. Insofern handelt es sich um einen wechselseitig aufeinander bezogenen Prozess von »Strukturverantwortung « und »Selbstorganisationspotenzial «.

12 Zur Strukturverantwortung der Führungskräfte - gehört ihre Vorbildfunktion für die Vermittlung und die Verkörperung der formulierten Vision. Hier ist »Leadership« gefragt.

13. Kopflastige Strategien des Veränderungsmanagements können Handlungsspielräume in der Organisation einengen. Es ist sinnvoll, gezielt mit der Kraft der Imagination zu arbeiten, weil dadurch die Mitarbeiterinnen und Mitarbeiter und die Führungskräfte ganzheitlich aktivierbar sind und damit eher Handlungsspielräume ausweiten oder neu erfinden.

14. Veränderungsmanagement wird von oben initi- iert. Auf der Führungsebene wird die Richtungsentscheidung getroffen: Funktionsoptimierung (Verbesserung der eingespielten betrieblichen Zirkularität) oder Innovation (Entwicklung einer neuen betrieblichen Zirkularität).

15 Wenn der Weg der Innovation gewählt wird, 1 . dann muss akzeptiert werden, dass die Leistungsfähigkeit der Organisation zunächst geringer werden kann. Das kann zusätzliche personelle Ressourcen und zusätzliche Finanzierung kosten. Enttäuschungen müssen gegebenenfalls bearbeitet werden. steht Schritt für Schritt eine neue Organisations- kultur. Sie ist sowohl Impuls als auch Ergebnis der Organisationsentwicklung. In ihr werden die Erfahrungen mit Komplexität, Instabilität, Fehlern und Toleranz integriert. Die am Beginn des Prozesses formulierte Vision spielt die Rolle eines Ordners in der neuen Zirkularität.

17 Ordner heißt hier: Die Vision sollte so formuliert - sein, dass sie eine Attraktion darstellt, eine positive Dynamik in der Organisation auslöst, damit die Mitarbeiterinnen und Mitarbeiter mehrheitlich sagen können: »Dafür will ich mich engagieren!« Veränderungsmanagement organisiert eine »lernende Organisation «. (2)

\section{Eine lernende Organisation entsteht durch}

- die Formulierung einer Vision

- die Bereitschaft der Mitarbeiterinnen und Mitarbeiter, persönliche Meisterschaft zu üben (»Selbstmotivation«)

- die Bewältigung mentaler Barrieren

- den Aufbau innerorganisatorischer Teams und Netzwerke

- die Beachtung einer Systemperspektive (die Organisation in ihrem Kontext)

10 Eine lernende Organisation ist prinzipiell irritier19 . bar. Insofern erhält sie sich eine Anpassungsfähigkeit sowohl nach innen als auch nach außen. Allerdings ist eine nur reaktive Instabilität oft nicht ausreichend. Notwendig ist häufig eine proaktive Instabilität im Sinne eines vorgehaltenen kreativen Freiraumes in der Organisation. Insofern kann es sinnvoll sein, in bestimmten Bereichen der Organisation durch bewusste Irritationen einen solchen Handlungsspielraum offen zu halten.

20 In der Regel ist es so, dass in einer lernenden Or• ganisation ausreichend analytische und ausreidie für die Überlebensfähigkeit der Organisation genügen. Aber dieses Wissen und die Fähigkeiten sind oft nicht verfügbar. Ein externer Berater ist selten klüger als die Klienten, die er berät. Diese Hypothese gilt zumindest für jene Berater, die sich an dem »Selbstorganisationsparadigma « orientieren. Solche Berater verstehen ihre Arbeit als »Mäeutik«, als Hebammenkunst. Sie beherrschen vor allem Techniken, die zur Selbstbefähigung der Klienten verhelfen.

21 Es gibt Bereiche in der Organisationskultur, die $\angle 1$. durch Selbstbefragung nur schwer zugänglich sind. Es handelt sich hier um geheime Spielregeln oder um Prozesse, die in der Alltagssprache als »Teufelskreise « bezeichnet werden. Hier kann es hilfreich sein, dass der Berater eine Modellierung solcher Kreisläufe anbietet - gewissermaßen als Reiseführer in die weitgehend unbekannten Reviere der Organisation.

2 Wenn man im Bild einer Personalisierung bleiben $\angle$. will, dann steht hinter geheimen Spielregeln und Teufelskreisen ein »Gegenspieler « zum »Ordner «, der in der Vision wirksam werden soll. Eine neue Zirkularität kann erst dann voll wirksam werden, wenn die Macht des Gegenspielers dadurch überwunden wird, dass die betreffenden Spielregeln und Kreise bewusst werden, ihre Wirksamkeit gezielt irritiert wird und sich neue Regelwerke in der Organisation entfalten können. 
23. Im Veränderungsmanagement wird ein kulturel- ler Wandel in der Organisation gestaltet. »Organisationskultur « ist insofern ein tragender Begriff (3), als er ein zentrales Kraftfeld in der Organisation bezeichnet, nämlich den Handlungsraum für die Mitarbeiterinnen und Mitarbeiter, der u. a. bestimmt ist durch

- nichtpersonale Ressourcen

- personale Ressourcen

- Aufträge

- Erreichbarkeiten

Bei der Veränderung der Organisationskultur müssen regelmäßig Verfahren der Selbstevaluation eingesetzt werden. Diese dienen in besonderer Weise der Entwicklung einer Selbstorganisationskultur.

24 In der Organisationskultur spielt die Art des Um- gangs miteinander eine große Rolle. Angesprochen ist Vertrauen als organisationale Essenz. Neben dem Personenvertrauen ist Systemvertrauen wichtig. Mit Systemvertrauen ist die Gewissheit angesprochen, dass innerhalb der Organisation fair getauscht werden kann, bei- dender Bedeutung. Neue Handlungsmuster entstehen oft im Kleinen, manchmal angestoßen durch schwache Ursachen. Aber sie tragen in sich schon Spuren der Attraktion. Wenn diese durch Reflexion sichtbar werden können, steigt ihre Attraktivität. Auf diese Weise wird deutlich, wie die neue Zirkularität eine neue Organisationskultur schaffen kann.

28 Es gibt unterschiedliche Formen der Ausdifferen28 . zierung von Organisationskulturen. Das hängt ab vom Entwicklungsstand der Organisation. Setzt sie eher auf Einzelintelligenz, auf Teamintelligenz oder auf Netzwerkintelligenz? Die »Intelligenzschwerpunkte « korrelieren mit dem Grad von Komplexität und Dynamik, auf den sich die Organisation einstellen muss. Entsprechend unterschiedlich sind auch Führungsaufgaben (anweisen, coachen, moderieren).

29 Organisationen, die auf Netzwerkintelligenz set29 . zen, sind darauf angewiesen, dass die Mitarbeiterinnen und Mitarbeiter ihre Selbstorganisationspotenziale in hohem Maße einbringen können. Strukturverantwortung bei den Führungskräften bedeutet, dass diese ihre Systemkompetenz entwickeln. In der Organisation selbst muss ein weitgehender Wertkonsens herrschen. Ebenfalls braucht die Organisation als Rahmen für die freie Dynamik

spielsweise im Verhältnis von Lohn und Leistung. Beide Formen des Vertrauens steigern die Tragbarkeit von Unsicherheit und sind deshalb hilfreich beim Umgang mit Komplexität.

25 Einen nicht unwesentlichen Beitrag zur Entwick25 .lung einer Vertrauenskultur spielt die Erhöhung der Vernetzungsdichte in der Organisation. Dies kann u. a. durch die Einführung einer Intranetplattform und durch Internetforen geschehen. Beides bietet eine gute Grundlage für organisationales Wissensmanagement. (4) Offener Wissensaustausch stärkt den Zusammenhalt. Darüber hinaus kann durch die Schnelligkeit, die im Intranet und in Internetforen ermöglicht wird, eher auf relevante Indikatoren für sich anbahnende Lösungen oder Probleme und Krisen geachtet werden.

26. Ein kreatives Wissensmanagement lebt von Span- nungen innerhalb der Kultur, die von Mitarbeiterinnen und Mitarbeitern gebildet wird. Spannungen entstehen regelmäßig zwischen den konservativen Kräften und den innovativen Kräften innerhalb der Organisation. Je konstruktiver gestritten werden kann und darf, desto besser ist das für eine Kultur des Wandels. Insofern sollten »Hofnarren «, »Vorkämpfer « und »Querdenker « nicht stigmatisiert werden. Es empfiehlt sich ein achtsamer Umgang mit ihnen. Sie erzeugen notwendige Differenzen, ohne die Innovation undenkbar ist.

27 Ohne Differenzen keine Innovation. Die Span- nungen, die durch Differenzen entstehen, können neue Handlungsmuster antreiben, wenn in einem System Selbstorganisation wirksam werden darf. In der Mitarbeiterkultur können sich selbst verstärkende Kreisläufe im Sinne einer neuen Zirkularität bilden. Diese müssen allerdings sichtbar werden können. Die Sichtbarkeit tritt durch Reflexion ein. Insofern sind Maßnahmen der Selbstevaluation in der Mitarbeiterkultur von entschei- verlässliche Regeln. Gemeinsam getragene Werte und vereinbarte Regeln bilden hier einen Stabilitätsrahmen für das Management von Instabilität. Unter diesen Voraussetzungen gelingt das Balancieren zwischen Stabilität und Instabilität.

30 Zum Balancieren zwischen Stabilität und Instabi• lität gehört auch phasenweise die Verlangsamung von Prozessen, vor allem wenn es darum geht, einen organisationalen Konsens im Veränderungsmanagement zu erzielen. Das dürfte bei der Erzielung eines Wertekonsens der Fall sein. Hier bieten sich dialogische Verfahren an. (5) Die Sozialwirtschaft befindet sich in einer Legitimationskrise. In solchen Zeiten ist die Besinnung auf die ihr eigenen Werte von zentraler Bedeutung.

(1) Kruse, Peter, Erkenntnisse von Chaos- und Selbstorganisationstheorie für die Gestaltung betrieblicher Veränderungsprozesse, in: Dieter Schwiering, Mittelständische Unternehmensführung im kulturellen Wandel, Stuttgart 1996, SchäfferPoeschel, S. 157-179.

(2) Peter M. Senge, Die fünfte Disziplin, Stuttgart 2003 (9. Auflage bei Klett-Cotta).

(3) Edgar H. Schein, Organisationskultur, BergischGladbach 2003 (Edition Humanistische Psychologie).

(4) BBJ Intern, Wissensmanagement bei BBJ, Berlin 2005, BBJ-Verlag, Internet: http://www.bbj.de).

(5) Martina und Johannes F. Hartkemeyer, L. Freemann Dhority, Miteinander Denken. Das Geheimnis des Dialogs, Stuttgart 1998 (Klett-Cotta). 